\title{
Surface Enhanced Raman Spectroscopy at Electrochemically Fabricated Silver Nanowire Junctions
}

\author{
Radhika Dasari and Francis P. Zamborini ${ }^{\dagger} * *$
}

${ }^{\dagger}$ Department of Chemistry, University of Louisville, Louisville, Kentucky 40292, United States

$\$$ Current Address: Department of Chemistry, Eastern Kentucky University, 521 Lancaster Ave, NSB 4126, Richmond, KY 40475, United States

* Author to whom correspondence should be addressed.

Supporting information
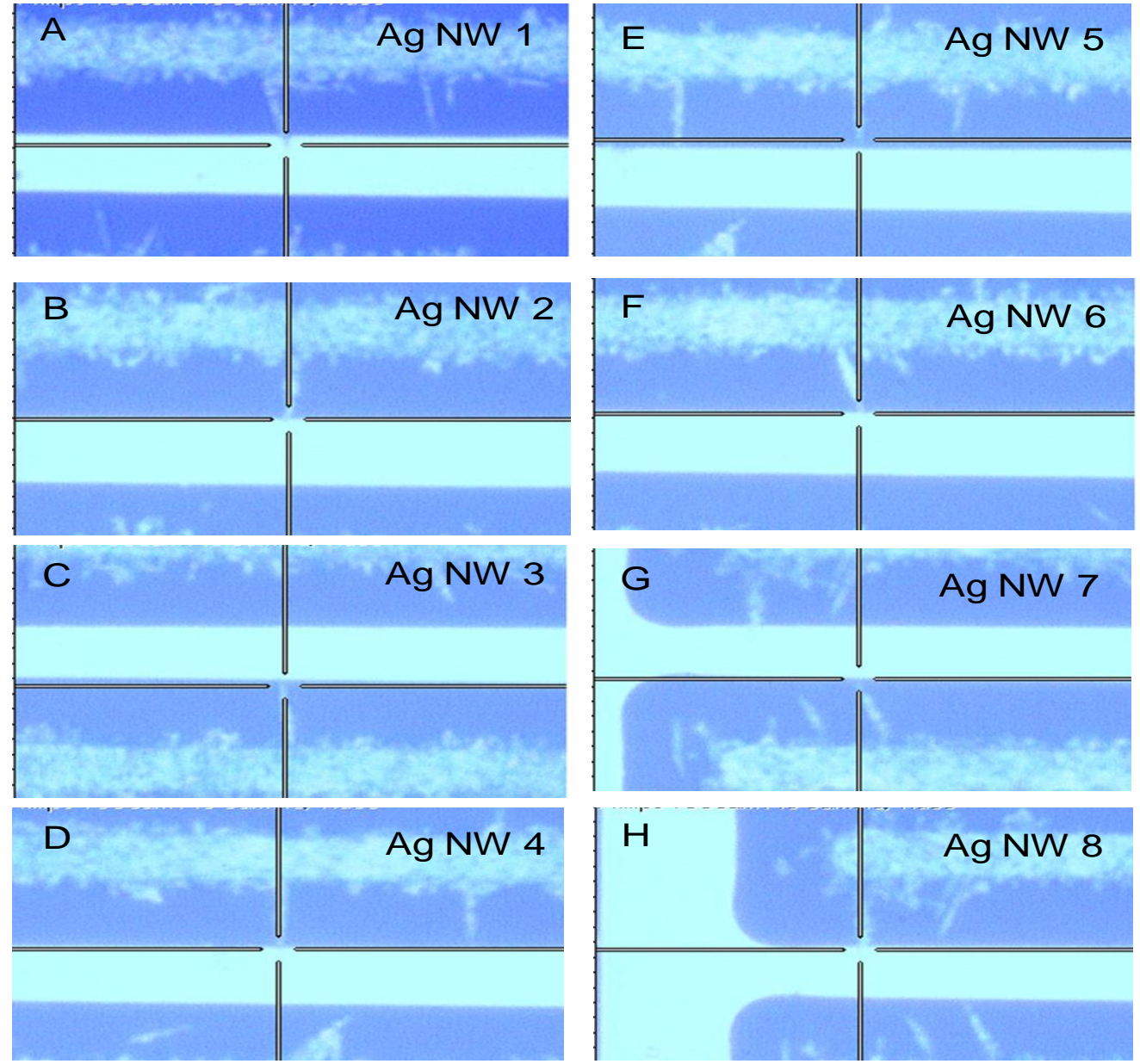

Figure S1. Optical images of different E1/Ag NW/4-ATP/E2 junctions numbered as 1 to 8 in the SEM image shown in Figure 1A. Cross hairs represent the area from where the Raman spectra shown in Figure $4 \mathrm{~B}$ in the main paper were collected. 


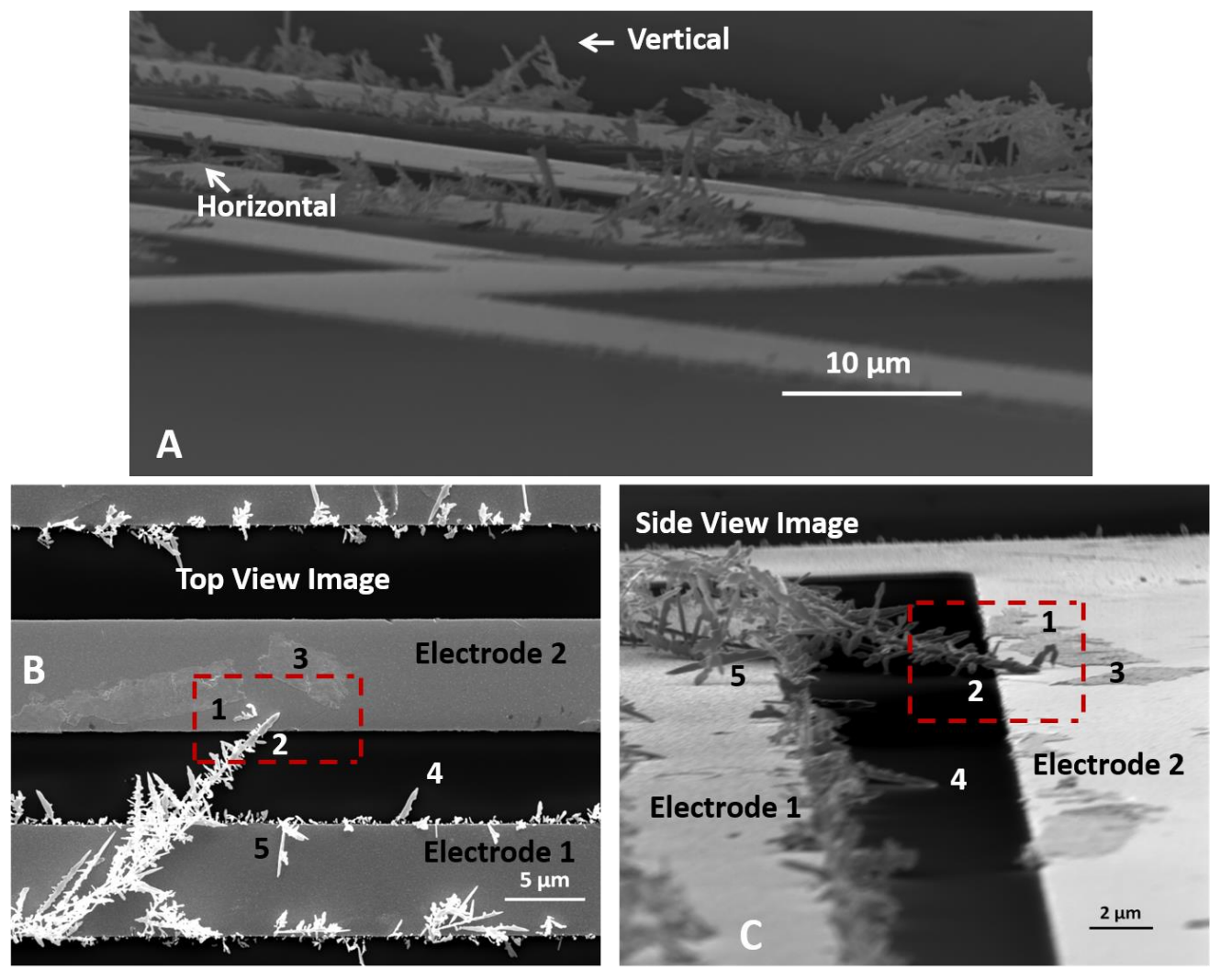

Figure S2. (A) Side view SEM images of an interdigitated array (IDA) device with different E1/Ag NW/4-ATP/E2 junctions showing that the Ag NWs grow horizontally and vertically. (B) Top view SEM of an Ag NW labeled as 2 and other features. (C) Side view SEM image of the same Ag NW as in B showing horizontal NW growth and good contact to Electrode 2. This results in a strong SERS signal for 4-ATP and good electrical contact. 
Table S1. Intensities of the Raman signal for different E1/Ag NW/4-ATP/E2 junctions within the same device and in different devices (I, II, III) for the $1071 \mathrm{~cm}^{-1}$ and $1576 \mathrm{~cm}^{-1}$ peaks (estimated to the nearest 10). The value in parentheses below the device number indicates the current displayed by the device at $1 \mathrm{~V}$. Enhancement factors were calculated for the Raman band at $1576 \mathrm{~cm}^{-1}$. The last row shows the total number of nanowires measured and range of values.

\begin{tabular}{|c|c|c|c|c|}
\hline $\begin{array}{c}\text { Device } \\
\text { (Current at } 1 \mathrm{~V} \text { ) }\end{array}$ & $\begin{array}{c}\text { Ag } \\
\text { Nanowire }\end{array}$ & $\begin{array}{c}\text { Intensity of } \\
1071 \mathrm{~cm}^{-1}\end{array}$ & $\begin{array}{c}\text { Intensity of } \\
1576 \mathrm{~cm}^{-1}\end{array}$ & $\begin{array}{c}\text { Estimated } \\
\text { Enhancement } \\
\text { Factor } \\
\left(1576 \mathrm{~cm}^{-1}\right)\end{array}$ \\
\hline \multirow{8}{*}{$\begin{array}{c}\mathrm{I} \\
\left(0.96 \times 10^{-9} \mathrm{~A}\right)\end{array}$} & 1 & 70 & 30 & $1.3 \times 10^{3}$ \\
\hline & 2 & 3480 & 5130 & $3.3 \times 10^{5}$ \\
\hline & 3 & 3350 & 2440 & $1.6 \times 10^{5}$ \\
\hline & 4 & 5930 & 6460 & $4.2 \times 10^{5}$ \\
\hline & 5 & 60 & 90 & $5.2 \times 10^{3}$ \\
\hline & 6 & 5600 & 9080 & $5.8 \times 10^{5}$ \\
\hline & 7 & 9510 & 21050 & $1.4 \times 10^{6}$ \\
\hline & 8 & 19480 & 28750 & $1.9 \times 10^{6}$ \\
\hline \multirow{4}{*}{$\begin{array}{c}\text { II } \\
\left(4.53 \times 10^{-9} \mathrm{~A}\right)\end{array}$} & 1 & 4020 & 3530 & $2.3 \times 10^{5}$ \\
\hline & 2 & 9210 & 5700 & $3.7 \times 10^{5}$ \\
\hline & 3 & 10180 & 9190 & $5.9 \times 10^{5}$ \\
\hline & 4 & 11770 & 12720 & $8.2 \times 10^{5}$ \\
\hline $\begin{array}{c}\text { III } \\
\left(13.0 \times 10^{-9} \mathrm{~A}\right)\end{array}$ & 1 & 36500 & 57820 & $3.7 \times 10^{6}$ \\
\hline Range & 13 wires & $60-36500$ & $30-57820$ & $1.3 \times 10^{3}-3.7 \times 10^{6}$ \\
\hline
\end{tabular}


Table S2. Intensities of the signal for the Raman band at $1576 \mathrm{~cm}^{-1}$ obtained from different junctions for pure 4-ATP and 4-ATP diluted with hexanethiol molecules as indicated (estimated to the nearest 10).

\begin{tabular}{|c|c|c|c|c|c|}
\hline \multirow[b]{2}{*}{ Sample } & \multirow[b]{2}{*}{ Ag NW } & \multirow[b]{2}{*}{ Pure 4-ATP } & \multicolumn{3}{|c|}{ 4-ATP:Hexanethiol } \\
\hline & & & $1: 9$ & 1:99 & 1:999 \\
\hline \multirow{8}{*}{ Sample 1} & Ag NW1 & 30 & 210 & 250 & 50 \\
\hline & Ag NW2 & 5130 & 680 & 1780 & 90 \\
\hline & Ag NW3 & 2440 & 2000 & 1660 & 250 \\
\hline & Ag NW4 & 6460 & 1690 & / & 50 \\
\hline & Ag NW5 & 90 & / & / & / \\
\hline & Ag NW6 & 9080 & / & / & / \\
\hline & Ag NW7 & 21050 & / & / & / \\
\hline & Ag NW8 & 28750 & I & I & I \\
\hline \multirow{7}{*}{ Sample 2} & Ag NW1 & 3530 & 1940 & 2490 & 230 \\
\hline & Ag NW2 & 5700 & 830 & 1820 & 170 \\
\hline & Ag NW3 & 9190 & 920 & 530 & 90 \\
\hline & Ag NW4 & 12720 & 1820 & / & 60 \\
\hline & Ag NW5 & / & 780 & / & 140 \\
\hline & Ag NW6 & / & 1490 & / & / \\
\hline & Ag NW7 & / & 1660 & l & / \\
\hline \multirow{5}{*}{ Sample 3} & Ag NW1 & 57820 & 450 & 1280 & 70 \\
\hline & Ag NW2 & / & 1700 & 380 & 130 \\
\hline & Ag NW3 & / & 1820 & 660 & 190 \\
\hline & Ag NW4 & / & / & 250 & 100 \\
\hline & Ag NW5 & / & I & 830 & 140 \\
\hline $\begin{array}{l}\text { Median } \\
\text { Intensity }\end{array}$ & & 6460 & 1575 & 830 & 115 \\
\hline
\end{tabular}

